Chapter 19

\title{
Small Molecule Screen in Zebrafish and HSC Expansion
}

\section{Eirini Trompouki and Leonard I. Zon}

\begin{abstract}
The zebrafish (Danio rerio) has emerged as a valuable model organism that is amenable for large-scale chemical and genetic screens. The ability of zebrafish to produce large quantities of synchronized, externally fertilized, transparent embryos makes them ideal for screens, which often are not possible in mammalian models. Signaling pathways important for hematopoiesis are well conserved between zebrafish and mammals, making many targets identified in zebrafish screens applicable to mammals. Hematopoiesis in zebrafish occurs in two waves: the primitive or embryonic wave and the definitive or adult wave. Definitive hematopoietic stem cells arise in the aorta-gonad-mesonephros region (AGM) and express conserved markers such as runxl and $c-m y b$ that allow for the detection of stem cells by whole-mount in situ hybridization (WISH). In this protocol, we will discuss a chemical screen in zebrafish embryos to detect compounds that expand or deplete hematopoietic stem cells (HSCs) in vivo. This type of screen represents a powerful tool to study HSCs in zebrafish.
\end{abstract}

Key words: Zebrafish hematopoiesis, HSCs: hematopoietic stem cells, Chemical screen, Chemical compound library, Chemoinformatics

\section{Introduction}

Zebrafish is an attractive model to study vertebrate hematopoiesis $(1,2)$ and has been used extensively for the study of many human hematological disorders (3). It shares characteristics of invertebrate models such as generation of large numbers of progeny, short generation time, and relatively small size that make it ideal for large-scale studies. In addition, hematopoiesis is well conserved between zebrafish and mammals (4). Zebrafish embryos are transparent and therefore ideal for fluorescent microscopy studies. Screens in whole organisms offer many advantages in comparison to tissue culture screens since the cells are in their physiological environment where it is easy to monitor in vivo in the context of adjacent tissues $(5,6)$. 
As in all vertebrates zebrafish hematopoiesis occurs in two waves, the primitive or embryonic hematopoietic wave and the definitive or adult wave. In zebrafish the primitive wave occurs in the intermediate cell mass (ICM), which gives rise to primitive erythroblasts, and the rostral blood island (RBI), which predominantly generates macrophages. The primitive erythrocytes are morphologically distinct from the adult erythrocytes and they enter the circulation around 24 h postfertilization (hpf). Several transcription factors, which are homologous to mammalian transcription factors, are expressed during the primitive wave of hematopoiesis and allow for the study of this event including $\mathrm{scl}$, flil, gata2, lmo2, and tifl $\gamma$. Definitive hematopoiesis gives rise to long-term HSCs, which maintain all mature hematopoietic lineages for the lifetime of the animal. This initially takes place in the aorta-gonad-mesonephros (AGM) region that in zebrafish is found in the ventral wall of the dorsal aorta around 36-40 hpf. Markers of the definitive HSCs include $c$-myb and runxl. These runxl+ HSCs then translocate to the kidney that becomes the major source of hematopoiesis during the lifetime of the zebrafish (7-9).

Large-scale screens are a major tool in zebrafish biology. Since HSCs are well defined in zebrafish it is expected that screens have been performed in order to find chemical factors and signaling pathways that can expand the HSC pool. In this chapter, we will describe a chemical screen in zebrafish that has been performed successfully and led to the discovery of chemical compounds that affect HSCs (10).

Zebrafish embryos, as already mentioned, are ideal for small molecule screens $(11,12)$. Chemical screens in zebrafish can lead to the discovery of molecules that affect certain pathways or developmental procedures, providing information that can finally lead to drug discovery. Zebrafish is also being used successfully to study potential safety liabilities of novel drugs (13).Chemical screens can provide information about toxicity of specific chemicals as well as tissue specificity since the screen is performed in whole organisms. A live organism may metabolize the chemical that is being tested, leading to activation of a pro-drug or an inactive metabolite. These effects cannot be studied in vitro and are studied much more accurately in vivo in a whole organism. Chemical screens can be used also for the discovery of modifiers in zebrafish mutants. If the mutation is embryonic lethal, these screens can be more labor intensive as heterozygous adults must be mated to generate mutant embryos. Since only $25 \%$ of the clutch will be mutants, fewer chemicals can be screened each week (14). Although small molecule screening in zebrafish is beneficial some potential problems have been identified. First, since the chemicals are added to the water, there is a question as to whether a given chemical can penetrate the chorion and permeate the 
embryo. Warfarin and dexamethasone are two examples of chemicals that have been shown to permeate into the embryo (15, 16). Colorful chemicals can provide direct evidence for the reagent permeability, for example, geimsa stain in a chemical library turns the embryos blue, while beta-carotene turns them yellow. Another major concern is the possibility that small molecules discovered by zebrafish screens will not be active in a mammalian system although some important drug-metabolizing enzymes studied in zebrafish seem to be conserved across species (17).

A small molecule screen to evaluate the effect of chemicals on HSCs has been performed successfully in zebrafish (10). The general design of this screen, described in detail below, is to collect thousands of zebrafish embryos, treat them with chemicals over the course of HSC development and perform whole-mount in situ hybridization for two HSC markers, runxl and $c-m y b$. Chemicals that augment or decrease HSCs in the AGM can be recognized, confirmed, and further tested in a mammalian system (Fig. 1).

Different screening procedures can be adapted depending on the desired result. For HSC screening in zebrafish, whole-mount in situ hybridization (see Note 1) (18) has been successfully employed as a screening modality. As mentioned earlier, two markers for the definitive stem cells of zebrafish are runxl and $c$-myb.(see Note 2)

\section{Materials}

\subsection{Embryo \\ Preparation}

1. E3 embryo medium: $5 \mathrm{mM} \mathrm{NaCl}, 0.17 \mathrm{mM} \mathrm{KCl}, 0.33 \mathrm{mM}$ $\mathrm{CaCl}_{2}, 0.33 \mathrm{mM} \mathrm{MgSO}_{4}$, and $10^{-5}$ methylene blue (see Note 3 ).

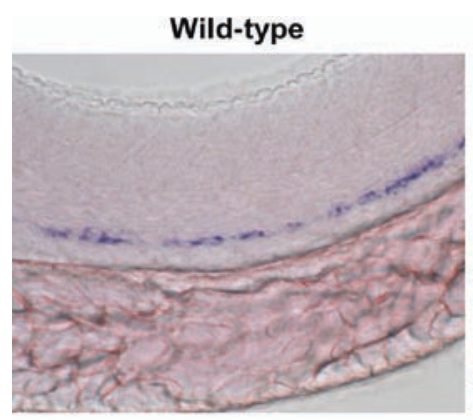

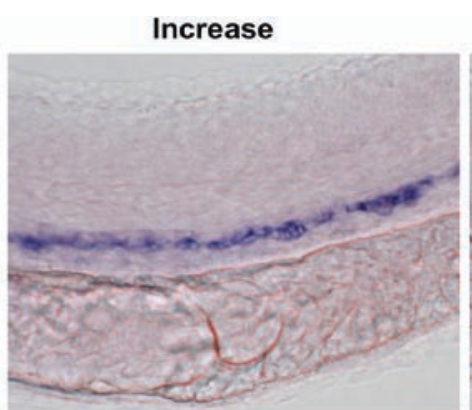

runx1/cmyb at $36 \mathrm{hpf}$

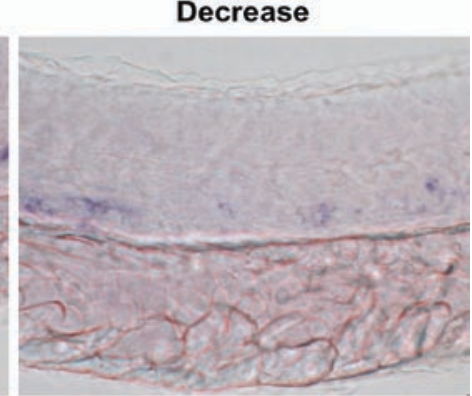

Fig. 1. Runx1/c-myb staining of zebrafish embryos at $36 \mathrm{hpf}$. The first picture represents the staining in a wild-type embryo. The second is a picture of an embryo treated with a chemical that increases HSC population whereas the third picture represents an embryo that has been treated with a chemical that decreases HSC population in the AGM. (The pictures in this figure are kindly provided by Trista E. North) 
2. 1,000× stock methylene blue.

3. Plastic pipettes and glass pasteur pipettes.

4. Tissue culture dishes.

5. Watchmaker's forceps (Dumont).

6. Incubator $28^{\circ} \mathrm{C}$.

7. Cages with dividers for timed embryo production.

2.2. Chemical Libraries and Chemical Screen
E3

E3+: E3 with 1\% DMSO and 1\% Pen/Strep

There are numerous chemical libraries commercially available. Chemicals are usually stored in 96 - or 384 -well plates at $-20^{\circ} \mathrm{C}$, $-80^{\circ} \mathrm{C}$, or at room temperature under $100 \%$ nitrogen. The libraries differ in the number and complexity of compounds as well as the purity of them. High purity of the compounds is essential since impurities can give false-positive or false-negative results on a screen. In the best available libraries the quality control of the compounds is performed by NMR. Chemical libraries can contain known or unknown chemical substances or they may be compiled from naturally occurring chemical compounds. below

Some examples of the available chemical libraries are given

- Libraries that contain biologically active compounds with defined biological activity. These libraries are the best solution for dissecting a biological pathway, most importantly, they offer good coverage over several biologically important pathways and the chemicals are usually provided in high purity. The disadvantage of some of these libraries is that usually they include only one or two chemicals per pathway. An example of this kind of library is the "ICCB known bioactive library" by Biomol.

- Natural product libraries. These libraries are the best choice when the screen is seeking a novel clinical or pharmaceutical product. Their major disadvantage is that they usually contain chemicals with low purity $50-80 \%$. That means that a possible active biochemical needs to be biochemically purified and retested in order to be verified, and to ensure that the impurities don't interfere with the action of the chemical. Many companies offer natural product libraries.

- Functional libraries. These libraries contain a collection of chemicals against a very specific target or with a specific action. Examples include a neurotransmitter library, or kinase inhibitor library. 
- Commercial libraries. These libraries are in general huge and contain tens of thousands of compounds. The advantage is maximal chemical diversity, while the disadvantage is that most compounds will not have any biological activity. These libraries are most useful when the screen is very high throughput.

Many companies offer the ability to create a custom chemical library (Chembridge http://www.chembridge.com/collected-screeninglibraries.html). The National Institute of Health offers a vast array of chemicals available for screens free of charge. Sigma Aldrich also offers a variety of chemical libraries, including the LOPAC1280 collection of pharmaceutically active compounds.

The libraries offered can be broad in order to cover a great extent of the available chemical compounds, or can be more biased according to the chemical identities of the compounds, the molecular weight of the compounds, the medicinal use, or other.

1. 96- or 48-well plates.

2. TECAN liquid handling robot to easily transfer chemicals from 96 to 48-well plates if desired (Tecan, Durham, NC) (see Note 4 )

2.3. Embryo Fixation, Dechorionation, Bleaching, Rehydration, and Proteinase $\mathrm{K}$ Treatment

\subsection{Hybridization}

1. Phosphate buffer saline (PBS): $0.14 \mathrm{M} \mathrm{NaCl}, 0.003 \mathrm{M} \mathrm{KCl}$, $0.002 \mathrm{M} \mathrm{KH}_{2} \mathrm{PO}_{4}, 0.01 \mathrm{M} \mathrm{Na}_{2} \mathrm{HPO}_{4}, \mathrm{pH} 7.2$.

2. 4\%PFA in PBS (see Note 5 ).

3. Pronase: $50 \mathrm{mg} / \mathrm{ml}$ in $\mathrm{E} 3$, aliquot, and store at $-20^{\circ} \mathrm{C}$ (see Note 6).

4. Bleach solution: $0.8 \% \mathrm{KOH}, 0.9 \% \mathrm{H}_{2} \mathrm{O}_{2}, 0.1 \%$ Tween in deionized water. Should be prepared fresh just before use (see Note 7).

5. PBST: PBS, 0.1\%Tween-20.

6. Proteinase $\mathrm{K}: 10 \mu \mathrm{g} / \mathrm{ml}$ in PBST, aliquot and store at $-20^{\circ} \mathrm{C}$ (see Note 8).

7. Watchmaker's forceps (Dumont).

8. 48-well mesh plates.

1. Deionized formamide: formamide should be aliquoted and stored at $-20^{\circ} \mathrm{C}$. Formamide is toxic and must be handled with care. (see Note 9).

2. Saline sodium citrate (SSC): $20 \times: 3 \mathrm{M} \mathrm{NaCl}, 300 \mathrm{mM}$ trisodium citrate, $\mathrm{pH}$ 6.0.

3. Hybridization solution (hybe solution): $50 \%$ formamide, $5 \times$ SSC, $0.1 \%$ Tween-20. Can be prepared and stored at $-20^{\circ} \mathrm{C}$.

4. PBST: PBS, 0.1\%Tween-20. 
2.5. DIG Staining (see Note 11)

2.6. Probe Preparation

2.7. Observation and Photography
5. Probe: dilute $100 \mathrm{ng}$ of probe in $100 \mu \mathrm{l}$ of Hybe solution.

6. $70^{\circ} \mathrm{C}$ oven.

7. Blocking solution: $2 \%$ heat-inactivated lamb serum $0.2 \% \mathrm{BSA}$ in PBST.

8. Anti-digoxigenin alkaline phosphatase (AP) Fab fragments (Roche) (see Note 10).

9. Antibody solution: $1 \mu \mathrm{l}$ of antibody in $5 \mathrm{ml}$ of blocking solution.

1. PBST: PBS, 0.1\%Tween-20.

2. Staining buffer: $100 \mathrm{mM}$ Tris-base, $\mathrm{pH} 9.5,50 \mathrm{mM} \mathrm{MgCl}$, $100 \mathrm{mM} \mathrm{NaCl}, 0.1 \%$ Tween-20. Add Tween-20 before use. The rest of the solution can be kept at $4^{\circ} \mathrm{C}$.

3. 4-Nitroblue tetrazolium (NBT) $(50 \mathrm{mg} / \mathrm{ml})$ in $70 \%$ dimethylformamide.

4. 5-Bromo-4-chloro-3-indolyl-phosphate (BCIP) $(50 \mathrm{mg} / \mathrm{ml})$ in $100 \%$ dimethylformamide.

5. Staining solution: dilute $35 \mu \mathrm{l}$ of $50 \mathrm{mg} / \mathrm{ml} \mathrm{BCIP}$ and $45 \mu \mathrm{l}$ of $50 \mathrm{mg} / \mathrm{ml} \mathrm{NBT}$ in $10 \mathrm{ml}$ of staining buffer.

1. Template DNA in appropriate vector.

2. Enzyme suitable for vector linearization.

3. DIG-labeling kit (Roche): DIG-11-UTP NTP mix, 5x Transcription buffer, SP6, T3 or T7 polymerase, RNAse inhibitor, RNAse-free DNAse, and RNAse-free $\mathrm{ddH}_{2} \mathrm{O}$.

4. RNeasy Mini kit or some other kit for purification of RNA.

1. Coverslips and microscope slides.

2. PBST.

3. Glycerol.

4. Microscope (we use Nikon Eclipse E600).

5. Camera (we use CoolPix 4500 4.0 Mega Pixel).

\section{Methods}

3.1. Embryo

Preparation
1. Set up the desired adult pairs overnight (male and female separated by divider) in mass mating cages $2-3$ females and 2-3 males per cage, or as single pairs.

2. Pull out the dividers to combine fish around $8-9$ a.m. to best synchronize fertilization timing. 
3. Collect different clutches into individual petri dishes using E3 water and methylene blue and stage the embryos to verify that they are synchronized.

4. At about 5 hpf clean out dead embryos and debris.

5 . When the embryos have reached the desired stage check them again to confirm that they are synchronized and pool all the embryos together (synchronized staging of the embryos is really crucial!).

6. For a screen in HSCs the treatment with the chemical can start as early as $\mathbf{5}$ somites stage or as late as 24 hpf depending on the specificity of the target desired. For different kind of screens it may be necessary to choose an earlier or a later stage. (see Note 12)

3.2. Chemical Libraries

1. Preparation of chemical plates.

2. Get the desired chemical library, for example, the ICCB.

3. Add $150 \mu \mathrm{lE} 3+$ into each well of the desired number of 96-well plates and add $1 \mu \mathrm{l}$ of chemical in each well. (see Note 13)

4. Transfer chemicals to 48 -well plates using Tecan robot or manually. Final volume is $300 \mu \mathrm{l}$ per well (drugs are used at an approximate concentration of $\sim 20 \mu \mathrm{M}$ ).

5. Pool synchronized embryos together to avoid clutch bias and remove as much of the E3 as possible. Scoop desired number of embryos with a small spatula and flick briskly to place embryos into each well. Embryo number can be adjusted according to the demands of each screen. For screens of wildtype embryos 5-10 embryos per well is an acceptable number. (see Note 14)

6. Incubate embryos in the chemicals at $28.5^{\circ} \mathrm{C}$. The time can be adjusted according to the demands of each screen. For an HSC screen embryos can be incubated from 5 somites stage till $36 \mathrm{~h}$ postfertilization. (see Note 15)

7. It is crucial that untreated, negative and positive controls (if available) are placed in every single plate of the chemical screen. The positive control can be used to verify the stopping point for the in situ hybridization staining and can validate the success of the whole procedure for each plate.

3.3. In Situ

Hybridization (see Note 16)

\subsubsection{Embryo}

Dechorionation, Fixation,

Bleaching and Rehydration, and Proteinase $K$

Treatment
1. Dechorionate the embryos. Dechorionation of embryos younger than 20 somites should be done manually with two fine watchmaker's forceps. The user can grab the chorion with the forceps and gently pull in opposite directions. The procedure should be performed under a dissecting microscope. After the dissection the yolk will appear dark but this will not affect the outcome of in situ hybridization. For an HSC screen the embryos will be collected at $36 \mathrm{hpf}$. In this 
case, the dechorionation can be performed with the addition of pronase. Add $10 \mu \mathrm{l}$ of $50 \mathrm{mg} / \mathrm{ml}$ pronase per well and leave for $5 \mathrm{~min}$. The chorion should be very easily detachable. Prolonged incubation with the pronase may result in embryo dissociation.

2. After dechorionation with pronase the embryos should be washed four times in E3 to remove pronase and the chemical.

3. Fix the embryos overnight in 4\% PFA.

4. After fixation, embryos that are $36 \mathrm{hpf}$ or older need bleaching, otherwise, the optical clarity of the embryos, a great advantage of zebrafish, will be severely diminished. To bleach the embryos remove PFA and wash twice with PBST twice. Put $300 \mu \mathrm{l}$ of bleaching solution in each well and incubate $10 \mathrm{~min}$ at RT for embryos $36 \mathrm{hpf}$ to $2 \mathrm{dpf}, 30 \mathrm{~min}$ for $3 \mathrm{dpf}$ embryos and 45 min for older embryos. Embryos after bleach are generally fragile so they must be handled with care.

5. Remove bleach and wash twice for 5 min with PBST.

6. Fix again in $4 \%$ PFA for at least $2 \mathrm{~h}$ at RT or overnight at $4{ }^{\circ} \mathrm{C}$ (PFA should be fresh or recently defrozen because embryos are fragile after bleaching).

7. Wash in $\mathrm{MeOH}$ three times for $5 \mathrm{~min}$ and fix in $\mathrm{MeOH} \mathrm{o} / \mathrm{n}$ at $4^{\circ} \mathrm{C}$ or $-20^{\circ} \mathrm{C}$ for at least $2 \mathrm{~h}$. Embryos can be stored at $-20^{\circ} \mathrm{C}$ for up to 2 weeks.

8. Rehydrate the embryos. From this step onward you can transfer embryos to 48 -well mesh screen trays, which facilitate the procedure or for use with the BioLane machine if available.

9. For each of the solutions mentioned use $500 \mu \mathrm{l}$ per well unless otherwise indicated. If you use the mesh 48 -well plates, use $50 \mathrm{ml}$ of solution per plate.

10. Wash once in MeOH:PBST 2:1 for $5 \mathrm{~min}$ RT.

11. Wash once in MeOH:PBST 1:2 for 5 min RT.

12. Wash four times in PBST for 5 min each RT.

13. Dilute $\mathrm{pK}$ in PBST to a final dilution of $10 \mu \mathrm{g} / \mathrm{ml}$ and add $200 \mu \mathrm{l}$ per well. This treatment can be hard for the embryos, so timing is really crucial. Another important point is that every batch of $\mathrm{pK}$ may differ slightly, as it is also possible for older versus newer stocks.

14. Suggesting timing for $\mathrm{pK}$ treatment

- Embryos younger that "bud": treat them for $30 \mathrm{~s}$ at RT.

- Early somitogenesis embryos: treat them for $1 \mathrm{~min}$ at RT.

- Late somitogenesis: treat them for $2 \mathrm{~min}$ at RT.

- Embryos 24 hpf: treat them for 5 min at RT. 
- Embryos 36/48 hpf: treat them for $10 \mathrm{~min}$ at RT.

- Zebrafish 5-6 dpf: treat them for $20 \mathrm{~min}$ at RT.

15. Wash once quickly with PBST. Washing should start immediately to prevent prolonged treatment with $\mathrm{pK}$. In mesh-48well plates the solution can be changed quickly. In the case of regular plates it is advisable to dilute the $\mathrm{pK}$ solution with PBST before trying to remove it, so that the time of exposure to $\mathrm{pK}$ is not prolonged.

16. Fix in 4\% PFA for $20 \mathrm{~min}$ at RT.

17. Wash four times in PBST for 5 min each at RT.

3.3.2. Hybridization

3.3.3. DIG Staining
1. Prehybridize the embryos in hybe solution at $70^{\circ} \mathrm{C}$ for $30 \mathrm{~min}$ up to $3 \mathrm{~h}$.

2. Dilute the probe in hybe solution at a final concentration of $\mathrm{lng} / \mu \mathrm{l}$ and prewarm it at $70^{\circ} \mathrm{C}$.

3. Replace hybe solution with hybe solution+probe $(250 \mu \mathrm{l})$ and incubate $\mathrm{o} / \mathrm{n}$ at $70^{\circ} \mathrm{C}$.

4. Next day prepare the wash solutions and preheat the appropriate ones at $70^{\circ} \mathrm{C}$. Use at least $500 \mu \mathrm{l}$ of washing solution for each well or $50 \mathrm{ml}$ if you are using the mesh plates.

5. Hybe $/ 2 \times \operatorname{SSC} 3: 1$ for $15 \mathrm{~min}$ at $70^{\circ} \mathrm{C}$.

6. Hybe $/ 2 \times \mathrm{SSC} 1: 1$ for $15 \mathrm{~min}$ at $70^{\circ} \mathrm{C}$.

7. Hybe $/ 2 \times \operatorname{SSC} 1: 3$ for $15 \mathrm{~min}$ at $70^{\circ} \mathrm{C}$.

8. $2 \times \mathrm{SSC}$ for $15 \mathrm{~min}$ at $70^{\circ} \mathrm{C}$.

9. $0.2 \times \mathrm{SSC}$ for $30 \mathrm{~min}$ at $70^{\circ} \mathrm{C}$.

10. $0.2 \times \mathrm{SSC}$ for $30 \mathrm{~min}$ at $70^{\circ} \mathrm{C}$.

11. $0.2 \times$ SSC/PBST $3: 1$ for $10 \mathrm{~min}$ at RT.

12. $0.2 \times$ SSC/PBST $1: 1$ for $10 \mathrm{~min}$ at RT.

13. $0.2 \times$ SSC/PBST $1: 3$ for $10 \mathrm{~min}$ at RT.

14. PBST for $10 \mathrm{~min}$ at RT.

15. Block the embryos in blocking solution $500 \mu \mathrm{l}$ per well for at least $30 \mathrm{~min}$ at RT.

16. Dilute the antibody $(0.15 \mathrm{U} / \mathrm{ml}, 1 \mu \mathrm{l}$ of antibody per $5 \mathrm{ml}$ of blocking solution) and incubate $\mathrm{o} / \mathrm{n}$ at $4^{\circ} \mathrm{C}$ (see Note 17 ).

1. Wash once quickly with PBST to remove the antibody solution.

2. Wash five times with PBST for $15 \mathrm{~min}$ RT on a rocker at $40 \mathrm{rpm}$.

3. Prepare staining solution and vortex. In $10 \mathrm{ml}$ of staining buffer add $35 \mu \mathrm{l}$ of BCIP stock $(50 \mathrm{mg} / \mathrm{ml})$ and $45 \mu \mathrm{l}$ of NBT stock $(50 \mathrm{mg} / \mathrm{ml})$. 
4. Wash once with staining buffer and then add $500 \mu$ l of staining solution and $500 \mu \mathrm{l}$ of staining buffer. This reaction is light sensitive so the plates must be kept in the dark. Monitor the reaction every $30-60 \mathrm{~min}$ to prevent high background staining. If $r u n x I$ and $c-m y b$ are used as probes for staining of the AGM staining should be stopped after several hours or it can be left $\mathrm{o} / \mathrm{n}$.

5. When the staining has reached the desired level remove the staining solution and wash multiple times with PBST. Stop the reaction by adding PFA $4 \%$, and store at $4^{\circ} \mathrm{C}$. Observe the embryos under a microscope for changes in the staining. In a screen, it is crucial that the same person evaluates all the plates of the screen so that there is consistency. The best solution is for two independent observers to judge the results and compare.

Probes for in situ hybridization can be made from a variety of vectors that contain a T3, T7, or SP6 polymerase site and can be linearized opposite that site with a restriction enzyme. In general, any in vitro transcription kit that adds digoxigenin-labeled UTP into antisense RNA can be used. The probe is crucial for the outcome of the screen so it is better to use a probe that gives a strict pattern and that is easily recognizable. The probes should be around $\mathrm{l} \mathrm{kb}$ in length but longer or shorter probes or partial probes can work equally well.

1. Digest $10 \mu \mathrm{g}$ of DNA with the appropriate enzyme. Digestion should be complete so longer than usual incubation times or even $\mathrm{o} / \mathrm{n}$ incubation may be needed.

2. Purify the DNA and measure the OD with nanodrop. Run a small sample on an agarose gel to check whether the digestion is complete. Dilute the DNA to a final concentration of $1 \mu \mathrm{g} / \mu \mathrm{l}$.

3. For the transcription reaction use

- $1 \mu \mathrm{g}$ of linearized DNA

- Transcription buffer (T3, T7 or SP6 polymerase) $2 \mu \mathrm{l}$

- NTP-DIG-RNA $2 \mu \mathrm{l}$

- $\quad$ RNAse inhibitor (35 units $/ \mu \mathrm{l}) 1 \mu \mathrm{l}$

- T3/T7/SP6 polymerase (20 units/ $\mu \mathrm{l}) 1 \mu \mathrm{l}$

- $\mathrm{ddH}_{2} \mathrm{O}$ to $20 \mu \mathrm{l}$ total volume

4. Incubate for $1 \mathrm{~h}$ at $37^{\circ} \mathrm{C}$.

5. Digest the DNA template by adding $1 \mu \mathrm{l}$ RNAse-free DNAse for $30 \mathrm{~min}$ at $37^{\circ} \mathrm{C}$.

6. Purify the RNA with RNeasy MINI kit from Qiagen or some other purification method. 
3.3.5. Observation and Photography

3.4. Chemoinformatics $(19,20)$
7. Measure the optical density of the RNA and run a gel to check for the integrity and the size of the RNA fragment. Aliquot probe and store at $-80^{\circ} \mathrm{C}$.

Observe the screen plates under the microscope for possible "hits." In case you have a "hit," the embryos should be photographed. Embryos that are 36 hpf are more easily photographed using a depression or a bridge slide to keep the embryos properly oriented. Coverslip slides by Nomarsky are also appropriate for taking pictures of the embryos. To make a bridge slide, stack and glue 3-4 coverslips on each side of a normal slide. The embryo can be put in the middle in a drop of glycerol, positioned as desired, then covered with another coverslip that bridges the two stacks. The embryos should be transferred from PFA 4\% to PBST and finally to glycerol so that they can be photographed (Fig. 1).

Performing a chemical screen can lead to the identification of many chemicals that produce a desired effect. It is possible that these chemicals have no known biological activity. In this case, there are tools to help the researcher to identify the potential activity of the unknown chemical by comparison to known chemicals. It is also possible to identify a known and FDA-approved drug that may exhibit the same activity as the unknown compound. The computational tool that gives the researcher these possibilities is chemoinformatics.

Chemoinformatics is the use of information technology to manage chemical information and solve chemical problems. Usually the chemicals that are included in a certain library, even though they may not have a known biological activity, are represented as molecules in different formats. The researcher can use these formats to compare to chemicals with known biochemical activity. Many types of formats exist. One of the most commonly used is the Simplified Molecular Input Line Entry Specification (SMILES) format (useful information about SMILES can be found in the following link http://www.daylight.com/dayhtml/ $\mathrm{doc} /$ theory/theory.smiles.html). Smiles is a very powerful format because it is simple and can be read rapidly by a computer. SMILES represent only the fundamental property of the molecule so they provide information that is not arbitrary. Another standard format is the Chemical Markup Language (CML) and the GROMACS file format family which is used in combination with the molecular simulation software GROMACS. Other formats are the CHARMM format, the Chemical File Format, the SYBYL Line Notation. It is also possible to convert between different formats. Two open source tools that can be used to convert between formats are the OpenBabel and JOELib.

The researcher can use these formats to search available chemical databases, using algorithms somewhat analogous to a 
BLAST search. Examples of such databases includePubChem, Wombat, MDL Drug Data Report (MDDR), Thor, CrossFire Beilstein, ChemBank, and many more. These repositories contain unique features but most of them enable the user to identify analog chemical substance to the one that they are interested in and they provide a link to 3D models and Safety Data Sheets as well as information about the purity of each chemical or the availability. MDL ACD provides also a number of suppliers for different chemicals. MDL ACD can be reached online via Discovery Gate where the researcher can execute structural or text-based searches. Most of the databases provide also links to literature and patents. The methods to query each database vary, but include the commonly used Tanimoto similarity score, which allows the researcher to determine how similar a given compound is to others in the literature with $\mathrm{n}$ dimensions by finding the angle between them. Alternatives include the SEA algorithm (http://shoichetlab. compbio.ucsf.edu/ keiser/sea/) or Tversky similarity (http:// chembank.broad.harvard.edu/welcome.htm) These tools, although not always successful, may sometimes point toward a class of similar compounds that can be tested for their ability to phenocopy the "hit" from a given screen.

\section{Notes}

1. In situ hybridization is an established readout for a screen in zebrafish. Another powerful readout is imaging of fluorescent fish, and the particular screen that we describe in this protocol could be performed using fish that express $c$-myb GFP(AGM staining), or c-myb GFP and lmo2-dsRED(AGM and vessel staining). The techniques for such a screen are still evolving. There are three main issues to contend with: (1) Sibling fish tend to exhibit significant variability in the strength of fluorescence. For that case, one should perform a prescreen and isolate some zebrafish pairs that produce embryos with similar fluorescent levels. (2) High-throughput fluorescent imaging needs to be rapid so that the embryos in the first well are at a similar stage to those in the last well screened on a given day. (3) Fluorescent visualization in typical 48-well plates tends to be of low quality, but this can be improved with the use of optical prism plates that allow for much improved image quality.

2. In situ hybridization on a large scale can be carried out using a robot. We use the Biolane HTI robot. This robot consists of two individual trays that can be each run independently with separate in situ protocols. The whole machine can be 
programmed to perform all the steps of the in situ hybridization. We usually use the robot from the rehydration step of the protocol until the washes, whereas the staining is performed manually. For the robot we use mesh plates provided by the same company (Hölle \& Hüttner AG).

3. E3 can be made and kept at room temperature as a $50 \times$ stock. Working stock is $1 \times$ and can be kept up to 2 weeks at RT. Methylene blue is usually added to $1 \times \mathrm{E} 3$ as a fungicide.

4. Chemicals can be transferred to the wells manually, but an automated system ensures a much faster and more accurate system avoiding pipetting errors.

5. Dilute PFA in PBS and heat the solution to $65^{\circ} \mathrm{C}$ to dissolve. If the powder is not completely dissolved add some drops of $1 \mathrm{~N} \mathrm{NaOH}$ to the solution till it is clear $(\mathrm{pH} \sim 7.5)$. PFA should be used fresh but it can be stored in aliquots and kept at $-20^{\circ} \mathrm{C}$. Do not freeze and thaw PFA.

6. Pronase is a mixture of a broad spectrum of proteases. Its activity is extended to both denatured and native proteins which can be digested into individual aminoacids. The mixture contains various types of endopeptidases (serine and metalloproteases) and exopeptidases (carboxypeptidases and aminopeptidases) as well as neutral and alkaline phosphatases. It is used here for the dechorionation of zebrafish embryos. It is obvious that prolonged exposure of embryos to pronase will affect their integrity so if the embryos appear fragile reduce the time of exposure to pronase.

7. Another common way to produce unpigmented embryos is the use of Phenylthiouria (PTU). PTU should be used to treat embryos younger than $24 \mathrm{hpf}$ but it has shown to cause delayed hatching, retardation, and embryo malformation in some cases although these effects are very mild at the working dose of $0.003 \%$ in fish water. PTU has also been shown to block thyroid hormone expression (21). For older embryos, bleaching is recommended.

8. Proteinase $\mathrm{K}$ is a broad spectrum serine protease. The predominant site of cleavage is the peptide bond adjacent to the carboxyl group of aliphatic, aromatic, or hydrophobic amino acids. Prolonged exposure to proteinase K may damage the embryos. The bleached embryos are especially fragile so a shorter treatment with proteinase $\mathrm{K}$ is recommended for bleached embryos.

9. Formamide lowers the melting point of nucleic acids and as a result the strands can be separated more easily. Formamide is highly corrosive in contact with skin or eyes and may be deadly if ingested. Formamide should never be handled without proper safety attire including gloves and goggles. 
10. Instead of digoxigenin other molecules can be also used in in situ hybridization probes, for example, fluoroscein or its derivatives such as fluoroscein isothyocyanate (FITC).

11. These solutions are toxic and light sensitive. The user should wear gloves while handling these solutions. BCIP and NBT are chemical compounds used for the sensitive detection of alkaline phosphatase (AP). BCIP is the alkaline phosphatase substrate and NBT acts as the oxidant. These two compounds together naturally form a purple precipitate but alkaline phosphatase act as a catalyst in this reaction and accelerates it approximately 1,000 fold. BCIP binds to the alkaline phosphatase active site but upon interaction with NBT, BCIP is released from the enzyme and the colorful precipitate is formed. In case the reaction is very slow, the addition of polyvinyl alcohol (PVA) accelerates the reaction by 20 -fold. In that case, the user should add PVA $10 \% \mathrm{w} / \mathrm{v}$ to the staining buffer that contains no Tween-20, boil the solution, let it cool down, and finally add Tween-20 (0.1\%) and use the solution. The use of PVA is recommended for less robust chromogenic substrates such as Fast Red. Levamisole can be used to block high endogenous alkaline phosphatase activity, and should be diluted in the staining buffer at a final concentration of $1 \mathrm{mM}$.

12. If a positive control for the screen is available, a time course with the known chemical should be performed in order to check the optimal initiation time for the screen (primary screen). Embryos should be treated at different stages and for different time points and a check should be made for the best possible readout. Earlier stages, for example, the start of gastrulation (50\% epiboly stage) may cause greater toxicity than choosing a stage after completion of gastrulation. The positive control is also essential to verify that the chemicals are able to penetrate the chorion since the treatment is performed before dechorionation. Important evidence regarding the penetrance of the chemicals is the fact that some chemicals are colored so they can be easily visualized to color the embryo. A negative control can also provide the time window, dose and different types or levels of nonspecific effects, but the positive control will also provide a picture of a potential positive regulator.

13. In a chemical screen it is generally too labor intensive to use different concentrations of the chemicals. This can result in toxicity from chemicals that should have been used in lower concentrations. It is also possible to miss a "hit" because a chemical needed to be more concentrated in order to have an effect. So, if the user has identified some chemicals that affect a certain pathway but missed others in the same pathway, we 
recommend retesting these chemicals and performing a doseresponse curve to find out the optimal dilution for each chemical.

14. The number of embryos depends on the kind of the screen. If the screen is performed on wild-type embryos, five embryos per well are generally enough to detect the desired result. When screens are performed on homozygous mutants that are embryonic lethal, the embryos must be generated by incrossing heterozygous adults so that only $25 \%$ of the total embryo population will be homozygous mutants. In this case, at least 20 embryos per well should be used to ensure enough numbers of the mutant embryos are tested with each chemical.

15. Pooling of chemicals is another suggestion in case large chemical libraries are screened. This is recommended for natural product libraries or commercial libraries that contain thousands of chemicals most of which will have no biological activity. Depending on the format of the library and the screening plate, the number of compounds per well can vary. The advantage of this method is that it reduces greatly the number of embryos requested for the screen. If the pooling strategy allows for each chemical to be represented twice in a plate, individual hits can be identified. The major drawback for the pooling strategy is the increased toxicity that can be very high, especially in libraries with known bioactive compounds.

Another issue is the incubation temperature. The suggested temperature can be modified according to the needs of the screen. Lower temperatures, for example, $25^{\circ} \mathrm{C}$ or $21^{\circ} \mathrm{C}$ will delay the development of the embryos if it is necessary.

16. As a general instruction for the whole in situ protocol, use large volumes of liquid for the washes. Smaller volumes are required for the Proteinase $\mathrm{K}$ treatment, bleaching, and the hybridization with the probe.

17. The antibodies can be absorbed against whole zebrafish embryos or zebrafish powder but we don't find this step necessary if we use dilution of the antibody at 1:5,000.

\section{Acknowledgments}

We would like to thank Trista E North for providing Fig. 1. We would also like to thank Trista E North, Jill L.O. de Jong, Xuining Le, and Richard M. White for providing their expertise in chemical screening and in particular Richard M.White for the 
chemoinformatics part of the protocol. Finally, we would like to thank Trista E North, Jill L.O. de Jong, and Richard M. White for careful review of the manuscript. Eirini Trompouki is supported by Jane Coffin Childs Memorial Fund.

\section{References}

1. Amatruda, J. F., and Zon, L. I. (1999) Dissecting hematopoiesis and disease using the zebrafish. Dev Biol 216, 1-15.

2. Dooley, K., and Zon, L. I. (2000) Zebrafish: a model system for the study of human disease. Curr Opin Genet Dev 10, 252-6.

3. Shafizadeh, E., and Paw, B. H. (2004) Zebrafish as a model of human hematologic disorders. Curr Opin Hematol 11, 255-61.

4. Galloway, J. L., and Zon, L. I. (2003) Ontogeny of hematopoiesis: examining the emergence of hematopoietic cells in the vertebrate embryo. Curr Top Dev Biol 53, 139-58.

5. Kari, G., Rodeck, U., and Dicker, A. P. (2007) Zebrafish: an emerging model system for human disease and drug discovery. Clin Pharmacol Ther 82, 70-80.

6. Lally, B. E., Geiger, G. A., Kridel, S., ArcuryQuandt, A. E., Robbins, M. E., Kock, N. D., Wheeler, K., Peddi, P., Georgakilas, A., Kao, G. D., and Koumenis, C. (2007) Identification and biological evaluation of a novel and potent small molecule radiation sensitizer via an unbiased screen of a chemical library. Cancer Res 67, 8791-9.

7. Davidson, A. J., and Zon, L. I. (2004) The 'definitive' (and 'primitive') guide to zebrafish hematopoiesis. Oncogene 23, 7233-46.

8. de Jong, J. L., and Zon, L. I. (2005) Use of the zebrafish system to study primitive and definitive hematopoiesis. Annu Rev Genet 39, 481-501.

9. Hsia, N., and Zon, L. I. (2005) Transcriptional regulation of hematopoietic stem cell development in zebrafish. Exp Hematol 33, 1007-14.

10. North, T. E., Goessling, W., Walkley, C. R., Lengerke, C., Kopani, K. R., Lord, A. M., Weber, G. J., Bowman, T. V., Jang, I. H., Grosser, T., Fitzgerald, G. A., Daley, G. Q., Orkin, S. H., and Zon, L. I. (2007) Prostaglandin E2 regulates vertebrate haematopoietic stem cell homeostasis. Nature 447, 1007-11.

11. Murphey, R. D., and Zon, L. I. (2006) Small molecule screening in the zebrafish. Methods $39,255-61$.
12. Mandrekar, N., and Thakur, N. L. (2008) Significance of the zebrafish model in the discovery of bioactive molecules from nature. Biotechnol Lett.

13. Barros, T. P., Alderton, W. K., Reynolds, H. M., Roach, A. G., and Berghmans, S. (2008) Zebrafish: an emerging technology for in vivo pharmacological assessment to identify potential safety liabilities in early drug discovery. $\mathrm{Br}$ J Pharmacol 154, 1400-13.

14. Murphey, R. D., Stern, H. M., Straub, C. T., and Zon, L. I. (2006) A chemical genetic screen for cell cycle inhibitors in zebrafish embryos. Chem Biol Drug Des 68, 213-9.

15. Jagadeeswaran, P., and Sheehan, J. P. (1999) Analysis of blood coagulation in the zebrafish. Blood Cells Mol Dis 25, 239-49.

16. Langenau, D. M., Ferrando, A. A., Traver, D., Kutok, J. L., Hezel, J. P., Kanki, J. P., Zon, L. I., Look, A. T., and Trede, N. S. (2004) In vivo tracking of T cell development, ablation, and engraftment in transgenic zebrafish. Proc Natl Acad Sci U S A 101, 7369-74.

17. Tseng, H. P., Hseu, T. H., Buhler, D. R., Wang, W. D., and Hu, C. H. (2005) Constitutive and xenobiotics-induced expression of a novel CYP3A gene from zebrafish larva. Toxicol Appl Pharmacol 205, 247-58.

18. Thisse, B., Heyer, V., Lux, A., Alunni, V., Degrave, A., Seiliez, I., Kirchner, J., Parkhill, J. P., and Thisse, C. (2004) Spatial and temporal expression of the zebrafish genome by large-scale in situ hybridization screening. Methods Cell Biol 77, 505-19.

19. Parker, C. N., and Schreyer, S. K. (2004) Application of chemoinformatics to highthroughput screening: practical considerations. Methods Mol Biol 275, 85-110.

20. Brown, F. (2005) Editorial opinion: chemoinformatics - a ten year update. Curr Opin Drug Discov Devel 8, 298-302.

21. Elsalini, O. A., and Rohr, K. B. (2003) Phenylthiourea disrupts thyroid function in developing zebrafish. Dev Genes Evol 212, 593-8. 